\title{
Leaving the Financial Nest: \\ Connecting Young Adults’ Financial Independence to Financial Security
}

\author{
Megan Doherty Bea* \\ Cornell University \\ Youngmin Yi \\ Cornell University
}

\begin{abstract}
Objective: This study examines variation in young adults' transitions to financial independence and the relationship between these transitions and financial security.

Background: Individuals on their families for substantial financial support well into early adulthood, even as young adults perceive independence as a key marker of adulthood. Given known variation in transitions to adulthood and unequal exposure to financial precariousness across social groups, the authors ask whether heterogeneity emerges with regards to the timing of financial independence and types of support received, and how differences in pathways to independence may matter for financial security later in young adulthood.
\end{abstract}

Method: The authors estimate group-based trajectory models of four indicators of financial independence for 1,719 young adults from age 18 - 27 using data from the 2005-2015 Panel Study of Income Dynamics (http://psidonline.isr.umich.edu/). These trajectories are then used to estimate predicted levels of financial security at the end of the study period, using logistic and linear regression analysis.

Results: Results show that paths to young adults' financial independence are best characterized by four types of trajectories: Consistently Independent (23\%), Quickly Independent (41\%), Gradually Independent (23\%), and Consistently Supported (13\%), with types and duration of support varying substantially across trajectories. The authors find that young adults experiencing trajectories characterized by lower levels of familial support also report higher levels of financial insecurity by the end of the survey.

Conclusion: The findings suggest that the patterning and timing of financial independence in the transition to adulthood has implications for financial wellbeing.

Keywords: inequality; intergenerational transfers; family economics; young adulthood

\footnotetext{
* The authors would like to thank Matthew Hall, Kelly Musick, and members of the Cornell Population Center for their thoughtful feedback on earlier versions of this paper. Please direct all correspondence to Megan Doherty Bea, Department of Sociology, Cornell University, 345 Uris Hall, Ithaca NY 14853; email: mad337@cornell.edu.
} 
In the United States, the transition to adulthood is a stage of the life course that is marked by change and uncertainty across many domains, including financial life. Two major economic trends are likely shaping the contours of the contemporary transition to financial independence. First, young adults today face significant financial burdens, in large part due to the rising costs of postsecondary education and housing, which affect the likelihood that individuals in this life stage are able to reach financial independence and, perhaps more importantly, financial security (Addo et al., 2016; Houle, 2014; Killewald, 2013). Second, young adults’ reliance on parental resources into early adulthood is increasingly common, even as they "achieve adulthood" in other ways, such as when starting one's own family or entering the labor market (Fingerman et al., 2015; Lee and Mortimer, 2009; Sironi and Furstenburg, 2012). This suggests that many may be delaying financial independence in efforts to manage this financial uncertainty.

It is increasingly common for contemporary young adults to rely on family, and research highlighting this trend underscores the delayed transition to financial independence for young adults via examinations of intergenerational monetary transfers and parental co-residence or “doubling up” (e.g. Sandberg-Thoma, Snyder, and Jang, 2015; Swartz et al., 2011). However, less is known about whether and how different types of financial support co-occur, who receives which types of support, and for how long, although the presence of broader heterogeneity in the transition to adulthood with respect to race/ethnicity, socioeconomic status (SES), geography, and other dimensions is well-documented (e.g., Gonzales, 2011; Hardaway and McLoyd, 2008; Osgood, Foster, and Courtney, 2010; Waters et al., 2011). Importantly, research focused on perceived delays in the attainment of financial independence does not connect such perspectives 
to financial security, making the implications of heterogeneity in young adults’ financial independence trajectories for their financial wellbeing unclear.

In this study, we use data from the 2005-2015 Panel Study of Income Dynamics (PSID) to answer three key questions. First, do all young adults become financially independent in the transition to adulthood, and, if so, what are their typical trajectories to independence? Second, how do these trajectories vary in their structure and who experiences which types of financial trajectories? Finally, how are these financial independence trajectories related to financial security in the transition to adulthood? We employ a rich descriptive longitudinal modeling strategy for our two-part analysis. We begin by estimating group-based trajectory models to identify patterns in young adults' transitions to financial independence as captured by multiple indicators that identify when and for how long young adults receive parental support for housing, tuition, and bills, and whether these types of support co-occur. Second, we explore the relationship between young adults' financial independence trajectories and their levels of financial security using logistic and ordinary least squares regressions.

We find significant heterogeneity in pathways to financial independence, and, importantly, that this variation matters for young adults' levels of financial worry and their likelihood of exposure to poverty. Relative to those who remain financially supported by family later into the transition to adulthood, young adults who do not, or cannot, rely on this support are more likely to be financially insecure in their late twenties. We argue that family support may serve as an important buffer against financial hardship for young adults, but that this option may be restricted to those who come from socioeconomically advantaged backgrounds.

\section{THEORETICAL BACKGROUND}

The Transition to Adulthood in a Context of Economic Uncertainty and Inequality 
Nearly all young adults consider financial independence to be a key marker of adulthood (Furstenberg et al., 2004; Hartmann and Swartz 2006; Hartnett et al., 2012). However, in today’s economy, this expected milestone may be difficult to achieve. The burden of complex decisionmaking regarding debt, equity, and finances more generally has shifted from financial experts to households and consumers (Fligstein and Goldstein, 2015; Hacker, 2006; van der Zwan, 2014). Job precariousness and low wages have become more prevalent within the United States, increasing income insecurity for many young adults (Danzinger and Ratner, 2010). At the same time, traditional milestones thought to characterize the transition to adulthood, such as the completion of one's education and establishment of an independent household and/or family, require significant financial investment. Rising housing costs and expansion of the pursuit of postsecondary education have made the assumption of major expenses and debt for housing and schooling normative and growing components of young adults' financial lives (Addo, Houle, and Simon, 2016; Joint Center for Housing Studies, 2016; U.S. Department of Education, 2016). Many young adults are facing significant financial burdens and uncertainty while navigating this economic landscape with varying levels of resources, skills, and knowledge—-both their own and that of their families — as they move toward financial independence (Silva, 2012).

For our cohort of interest, coming of age in the mid-late 2000s, navigating the transition to financial independence has coincided with the 2008 - 2009 recession, which contributed to heightened economic uncertainty for many American households. The crisis compounded financial burdens through layoffs, foreclosures, and losses related to invested wealth (van der Zwan, 2014). Young adults were especially affected by rising unemployment during and following the recession. In fact, during this time, those aged 16-24 years experienced the most precipitous employment losses of all age groups of U.S. workers (Engemann and Wall, 2009). 
These economic shifts, as well as the increased complexity of financial decision-making in early adulthood, make young adults' capacity to meet their financial needs less certain.

\section{Family Assistance in the Transition to Adulthood}

For the families that can afford it, this means that within-family transfers have become critical resources for their maturing children (Swartz, 2009). Given the previously-discussed growth in costs associated with the transition to adulthood, there is reason to believe that parental support is a welcome resource for many young adults navigating this transition. Indeed, over half of individuals entering young adulthood in the mid-late 2000s received at least some financial support from their families (Wightman, Schoeni, and Robinson, 2012). Some scholars refer to the expansion of familial financial support in early adulthood as indicative of a "delay" in financial independence and the transition to adulthood more broadly (Fingerman et al., 2012; Hartnett et al., 2012; Sironi and Furstenberg, 2012), with concerns about how extended financial support may negatively impact family well-being and individual development (Furstenberg 2010; Mortimer et al. 2016). However, studies that highlight heterogeneity in family dynamics provide alternative perspectives. For one, rather than marking a delayed transition, extended support may be indicative of families' efforts to provide a financial safety net during a life stage marked by uncertainty (Fingerman et al., 2015; Johnson and Benson, 2012; Maroto, 2017; Swartz et al., 2011). Additionally, families may have different norms and expectations for parent-child exchanges of monetary, affective, and other resources (Hamilton 2016; Moen and Wethington 1992; Swartz 2009), which may include some within-family differences in these practices across children (e.g. Suitor and Pillemer, 2006, Swartz, 2009).

These delays in financial independence may be relatively common, but there may be socioeconomic and demographic variation in the ability to deploy this financial "strategy" of 
extended support as well as in expectations around family dynamics related to money. Young adults coming from families with more financial resources tend to receive more financial assistance from their parents (Fingerman et al., 2015; Swartz et al., 2011; Wightman, Schoeni and Robinson, 2012). Further, these young adults can often rely on this support later into the transition to adulthood, relative to their less advantaged peers (Johnson and Benson, 2012; Fingerman et al., 2015). In contrast, young adults and adolescents from lower SES backgrounds and of racial/ethnic minority groups are more likely to be responsible for supporting not only their own needs but those of their families (Newman, 1995; Silva, 2012).

\section{Financial Independence versus Financial Security}

Just as there are demographic and socioeconomic differences that are associated with receipt of familial financial support, the efficacy of these resources in mitigating young adults' economic hardship varies with their backgrounds, characteristics, and the life circumstances that shape their early adult years. In some cases, earlier or "timely" financial independence may be beneficial to both the young adult and their family by allowing the young adult to adhere to age norms and releasing their parents from financial responsibilities to their children as they age. Yet in other cases, a similarly-timed transition may result in a premature launch of sorts. In this situation, an individual may be left unprepared or unable to shoulder ongoing and new financial burdens and may lead to later financial distress for the family should the young adult need to turn back to them for support unexpectedly.

Even if a family is willing and able to support a child, both the amount of support available and the returns to the that financial investment may vary across socioeconomic and demographic groups. For example, financial investment in college education and associated expenses, though potentially requiring an extended reliance on family support, may correlate 
with later financial well-being through returns in the labor market. However, the same human capital investment may not translate to the same returns for those without the access to the same support for tuition and school-related expenses. In fact, research indicates that debt burdens are more severe and, in the case of student debt, less definitively tied to later financial returns for minorities and young adults of lower SES, in part because familial financial support to offset debt is not as likely to be available (Addo et al., 2016; Houle 2014; Killewald, 2013). Given that these individuals are of the same groups that are overrepresented in low-wage and precarious work (e.g. Browne and Misra 2003) and more likely to face discrimination in the labor market (e.g. Hardaway and McLoyd, 2008; Pager 2003), their economic disadvantage may be compounded by differences in the types of family support received. Further, although more economically advantaged young adults may be able to put family support towards future investments that facilitate the transition to adulthood, such as the purchase of a home or for college tuition, those who are less advantaged are more likely to need family support simply to make ends meet. Thus, the relationship between receipt of financial support from family and financial security for young adults remains unclear.

With this in mind, we argue that a general measure of financial independence—-such as a single measure of monetary value of support received — provides an incomplete picture of young adults' financial wellbeing in the context of the contemporary economy. A comprehensive view of young adults' financial health must examine the independent patterning as well as connections between different types of financial support, independence, and security, agnostic about whether financial independence is "good" or "bad."

\section{Hypotheses}


In this study, we expect our results to confirm prior work documenting socioeconomic variation in families' likelihood of providing support to their children through this extended life stage, with individuals from more resourced families more likely to receive support at all and for a longer duration in the transition to adulthood. However, the degree to which these trends are likely to hold across different types of financial support and for different social groups, as well as the implications of these trajectories for young adults' financial security, all remain empirical questions central to our understanding of the financial lives and broader wellbeing of young adults in this critical stage of the life course.

As such, we have several hypotheses about the pathways to financial independence that will emerge from our analyses. We expect to see clear distinctions in the trajectories of financial independence. On one end of the spectrum, we expect to have a group of individuals who byand-large never receive support from their families because their families are more likely to be resource-constrained. On the other end, we expect to observe a group of individuals who receive consistent parental support across this life stage. We also expect that many individuals will fall in the middle, gradually transitioning away from family support as they age into their late twenties.

We also anticipate that different types of family support are mobilized (and retracted) at different times during this life stage. A multidimensional approach to the study of financial independence allows us to tease out important distinctions that might be masked by single-item measures of financial independence and support. Prior research finds broad SES differences in the receipt of financial support from families. However, as noted above, most studies look at either the monetary amount and/or the frequency of transfers provided to young adults rather than examining variation in different types of support. To this end, our study identifies whether, 
for what, and how late into the transition to adulthood individuals draw on familial financial support, and how this relates to their socioeconomic and demographic backgrounds.

Finally, anticipating that attainment of financial independence may not necessarily translate to financial security, we examine how different financial support trajectories are associated with young adults' financial security. We anticipate that there will be clear associations between the heterogeneous pathways to financial independence and end-oftrajectory financial security. Notably, we expect that early attainment of financial independence may be associated with lower levels of financial security, relative to later financial independence transitions experienced by peers who can rely on family support for longer. This is because family support may serve as a buffer to any financial uncertainty and/or hardship that young adults may face and may also in itself be reflective of a young adult's socioeconomic position.

\section{Data, Measures, AND Methods}

\section{Data and Sample}

We use data from the Panel Study of Income Dynamics (PSID), which has followed a nationally-representative sample of U.S. households since 1968. These data are available to the public and furnished by the Survey Research Center at the Institute for Social Research at the University of Michigan at Ann Arbor (Survey Research Center, 2017). For our analysis, we use the core biennial 2005-2015 individual, family, and Transition into Adulthood Supplement (TAS) files of the PSID. The TAS data provide detailed information on adolescent and early adult experiences of the children of PSID adults, including those related to financial responsibility and support. The longitudinal nature of these data, the relevance of the PSID to topics of personal finance, and the availability of detailed information about respondents' lives in 
early adulthood make these data especially appropriate for examination of changes in young adults' financial responsibility, receipt of familial financial support, and financial security. Between 1997 and 2007, children of the core PSID families participated in the PSID’s Child Development Supplement (CDS), designed to capture detailed information about children's home and developmental contexts and experiences. As these child participants age, they become eligible to join the TAS sample. If a young adult was a CDS respondent and is either 18 to 27 years old or is 17 years old and has finished high school, they are eligible to participate in the TAS. 2,893 young adults completed at least one wave of the 2005-2015 TAS.

Although 2,893 individuals appeared at least once in the TAS, our interest in examining within-person changes in receipt of familial financial support and self-reported financial responsibility and security requires further restriction of the sample. We use group-based trajectory models with linear and quadratic specifications, which require at least three repeated measurements of our measures of financial responsibility and support. We lose 1,112 cases, or $38 \%$ of the TAS, respondents to this empirical restriction. Of these 1,112 cases, 738 individuals (68\% of dropped cases) entered the survey in 2013 or 2015, which precluded them from the possibility of providing three waves of data. We dropped the remaining 374 of 1,112 cases due to sample attrition or survey non-response that resulted in their provision of fewer than three waves of data. We drop an additional 62 respondents (2\% of the TAS) who were missing information on the key conditioning covariates for our estimated trajectory models—parental education, race/ethnicity, sex, high school completion, and an indicator of whether the respondent entered the TAS after the Great Recession (in or after 2009)—leaving us with an analytic sample of 1,719 individuals (6,473 person-year observations). 
The respondents dropped from our sample do not differ from our analytic sample in terms of race/ethnicity or parents' highest level of educational attainment. They do differ statistically from our analytic sample with respect to whether they had completed high school or equivalent level of education at baseline (70\% in the dropped sample vs. $80 \%$ in the analytic sample, $\mathrm{p}<$ .001 ) and sex (46\% female vs. $51 \%$ female, $\mathrm{p}<.01$ ). The analytic sample also has a higher average number of years observed and average age, which is expected due to the exclusion criteria necessary to estimate the trajectories, described above.

Measures of financial responsibility and support. For our multidimensional approach to financial independence we consider four self-reported measures: three dichotomous measures of receipt of familial financial support and one continuous scale of financial responsibility. The first three measures report receipt of: 1) monetary support for bills or other routine expenses; 2) housing assistance, either in-kind (respondent lives with a parent/relative) or assistance with mortgage or rent payments; or 3) funds for school tuition at any point in the last year. Our measure of housing assistance accounts for the fact that some families use co-residence as a way to extend resources, potentially offsetting non-negligible living expenses without paying for other housing (Mykyta and Macartney, 2012; Pilkauskas et al., 2014). We also anticipate that although our measure of tuition support explicitly asks about postsecondary education tuition, the actual expenses respondents include in that category may also include room and board or other non-tuition expenses (e.g., meal plans, activity fees), which have grown alongside tuition (López Turley and Desmond, 2011). An advantage to using dichotomous reports rather than continuous measures of support received is that the former are less vulnerable to common issues of underreporting observed in income-related survey items (Kim and Tamborini, 2012). It is possible that there is still underreporting of receipt of financial support if respondents elect to 
classify a one-time transfer as a "gift" but not financial support. However, the TAS subsequently asks a separate question about inheritance and large gifts, distinguishing between these types of transfers, which mitigates this concern. We do not use the inheritance/large gifts survey item in this analysis due to the small number of individuals reporting receipt of such gifts (less than $9 \%$ of the full TAS sample).

Our final measure of financial independence is a five-point Likert-type scale of selfassessed financial responsibility constructed by PSID researchers. This measure is generated from four questions that ask whether the respondent 1) earns their own living, 2) pays rent, 3) pays bills, and 4) manages their own money. A response of "1" indicates that the individual is not responsible for any of the above and " 5 " indicates that they are entirely responsible for all of these aspects of their financial life.

Measures of financial security and wellbeing. To examine how these trajectories relate to financial security, we analyze two measures of financial security, a self-reported measure of worry about money and poverty status. Worry about money is reported on a continuous scale ranging from 1 to 7 , with higher values indicating more acute stress and concern. An individual is classified as being in poverty if their family income falls below $100 \%$ of the appropriate family size and composition-specific Census needs standards for that year. By using both measures, we capture subjective financial stress that young adults might feel as well as a more “objective” measure of material insecurity.

\section{Analytical Approach}

We use group-based trajectory analysis (GBTA) to provide a detailed picture of young adults' receipt of financial support from family and their own sense of financial responsibility as they move through this stage of the life course. GBTA uses maximum likelihood estimation to 
identify specific subgroup trajectories of, in our case, financial independence and receipt of financial support (Nagin, 2005). This method uses observed characteristics to identify latent classes (trajectories) and estimate probabilities of membership in each estimated trajectory, assigning each case to the trajectory type for which they have the highest likelihood of membership (Jones and Nagin, 2013; Nagin, 2005). Although there is some disagreement about whether and when to use certain types of trajectory models (see, e.g. Warren et al., 2015), we elect to use GBTA because it enables us to use a more inductive approach, identifying ex-post the factors that characterize individuals within estimated trajectories, rather than fitting trajectories to social groups pre-defined by the researcher, which could force the identification of trajectories that are not as likely to be found in the population.

Conditional group-based trajectory analysis. Because prior literature on intergenerational transfers shows that receipt of family financial support, and modal experiences in the transition to adulthood more generally, are associated with socioeconomic and demographic factors, we condition our models on five time-invariant risk factors, measured at time of entry into the TAS: race, sex, parental education, the young adult's own educational attainment, and a recession indicator of whether the respondent entered the TAS after the Great Recession (after 2009). This also means that the estimated probabilities of group membership are conditional probabilities, similar to how covariate adjustment in regression analyses impacts coefficient estimates and their interpretation. Parental education is employed as a proxy for young adult's SES and is measured as parents' self-reported education level in years; if two parents' education levels are reported, the higher of the two is used. Supplemental analyses that use total family income in lieu of education level produce virtually identical results (not shown). The young adult's own educational attainment is defined by whether the young adult has received a high school degree 
or equivalent education. Race is a categorical variable composed of the following mutuallyexclusive groups: White, Non-Hispanic; Black, Non-Hispanic; and Other. Sample size limitations restrict us from using more detailed racial/ethnic categories. Sex is a dichotomous measure, with those identified as male serving as the reference group.

The trajectories align with the biennial nature of the PSID using 2-year age intervals as the time metric (17-19, 20-21, 22-23, 24-25, and 26-27 years). We fit linear and quadratic specifications to account for the possibility that the relationship between age and financial responsibility/receipt of support is non-linear. We first fit models separately for each of our four financial independence measures (logistic GBTA models for family assistance with housing, bills, tuition, and a censored normal model for self-assessment of financial responsibility) to identify the best-fitting models to use for our analysis. A detailed discussion of our model selection procedure is available in the Methodological Appendix. Because each of the four financial independence processes are occurring simultaneously, we then fit a multi-trajectory model that jointly assesses the four measures by linking together the best-fitting conditional trajectories for each. This model accounts for each component of financial independence and their joint effects by taking into account each single-item trajectory and the probabilities of trajectory group membership within and across the four financial independence items (Nagin et al., 2016). Ultimately, this model identifies groups of individuals sharing similar trajectories across all four financial independence measures. Appendix B reports the coefficients and standard errors for each risk factor conditioning the model.

Assembling profiles of individuals within trajectories. We next classify individuals into multi-dimensional trajectory groups using maximum posterior probabilities. Posterior probabilities are calculated following model estimation and identify each individual's conditional 
probability of belonging to each trajectory group given the individual's observed behavior in each of the time periods (Nagin 2005). The group for which an individual has the highest conditional probability of membership—i.e. the trajectory “type” that best matches individual's own trajectory—is the group to which we assign the respondent. We use summary statistics to examine group differences in the five risk factors used to condition models as well as end-oftrajectory characteristics that reflect key milestones in the transition to adulthood: completion of any post-secondary education, completion of a four-year college degree, possession of student debt, partnership status (married, not married and living with a romantic partner, single), parental status, and employment status. The summary statistics are weighted to account for differences in individuals’ maximum posterior probabilities of being assigned to a group given trajectory group size (Nagin, 2005) as well as the sampling design of the PSID-TAS, which includes an oversample of lower-income households (Survey Research Center, 2015).

Linking trajectories to financial security. The final stage of analysis uses regression analysis to estimate subjective and objective measures of financial security as functions of financial independence trajectory group, taking family characteristics (partnership and parental status) and employment status into account. These estimates are weighted to account for individuals' conditional probabilities of membership in each trajectory group, estimated within the group-based trajectory models fitted within the first stage of our analysis.

\section{RESULTS}

A statistical socioeconomic and demographic description of the analytic sample is presented in Table 1. Most respondents have obtained a high school degree by the time they have entered the TAS sample (80\%). Their parents have an average of 14 years of schooling. Just over half of the sample is female (53\%) and the majority of the sample is non-Hispanic White (63\%); 
$17 \%$ of the sample is Non-Hispanic Black. Table 1 also presents information about the period of the life span for which we observe our sample; the average age of our sample at first interview is 18.6 years, and their average age at last observation in the TAS is 24.9 years. The average individual is seen intermittently for approximately 6.3 years of their life.

[Insert Table 1 about here]

Summary statistics of the outcome measures of interest at individuals' first and last observations are also presented in Table 1. As expected, average levels of self-reported financial responsibility are higher at the last time observed (moving from 3.5 to 4.5 on a scale from 1 to 5 ) while proportions reporting receipt of parental or familial financial support for bills and expenses, housing, and tuition are lower later in the study period. Looking at financial stability, individuals' self-reported worry about money remain relatively stable over the transition to adulthood and on average reflect a stable moderate amount of worry (3.8 and 3.7 out of 7 at first and last time observed, respectively) while their likelihood of being in poverty increases from about $9 \%$ to $13 \%$. This statistical description suggests that over the transition to adulthood, on average, individuals are indeed taking on more responsibility for their finances and becoming financially independent from their parents, though this may be occurring as financial insecurity is increasing. These averages, however, cannot speak to the specific trajectories that these young adults experience. As such, we now turn to the results of our conditional trajectory models, which provide a more fine-grained view of financial independence over this life stage.

\section{Multi-Dimensional Financial Trajectories}

Receipt of different types of support—for bills, housing, and tuition —as well as young adults' own perceptions of their assumption of financial responsibility are all interlinked but individually dynamic components of their financial lives in the transition to adulthood. Thus, 
individuals who receive one type of support may be systematically more (or less) likely to receive another type of support, and a comprehensive understanding of financial independence trajectories should acknowledge these life course experiences in concert. Our analysis centers on a multi-dimensional conditional group-based trajectory model that identifies typical trajectories for the aforementioned bundle of markers of financial (in)dependence.

The final model converges on four distinct trajectory groups (see Figure 1). The risk factors included in the model (parents' education, own secondary education attainment, race, sex, and post-recession entry) are, with the exception of sex, significant predictors of group membership, indicating that financial independence trajectories are strongly associated with these socioeconomic and demographic factors. Although sex does not emerge as a significant risk factor for group membership, we retain this measure because of gendered patterns in family financial transfers and the transition to adulthood shown in prior research. We use the maximum posterior probabilities from the estimated model to identify the best-fitting group for each individual. In the best-fitting model, the average posterior probabilities of group membership for each of the four trajectories range between 0.85 and 0.91 , compared to a recommended threshold of 0.70 for an acceptable minimum average posterior probability (Nagin, 2005). This indicates that the trajectory groups to which individuals are matched, though probabilistically determined, are highly likely to reflect their observed financial experiences.

[Insert Figure 1 about here]

Figure 1 presents the model results that show group trajectories over time, across the individual financial independence measures. Financial support trajectories differ significantly across the four groups, which we label as Consistently Independent, Quickly Independent, Gradually Independent, and Consistently Supported. 
Consistently Independent: From the outset, young adults in this first group are fairly independent for two of the three familial support items and stay independent over this life stage. Only about $25 \%$ of individuals receive support for bills across this stage of the life course, and almost no one receives tuition support. However, well over half of individuals report receiving in-kind or monetary support for housing for much of this life stage. In supplemental analyses (not shown), we find that, of the individuals receiving support in this group, the vast majority were receiving in-kind support, with only about $5 \%$ receiving help with their own rent (as opposed to assistance via coresidence), even in early years. Despite receipt of housing support, this group consistently reports high levels of financial responsibility (about a 4 on a 1-5 scale), suggesting that they take responsibility for much of their own finances. $23 \%$ of individuals ( $\mathrm{N}=$ 397) in our sample are best matched to this group.

Quickly Independent: This is the largest group and contains about $41 \%$ of the individuals in our sample ( $\mathrm{N}=711$ ). Many individuals in this group start out dependent upon family for housing, and to a lesser extent, for bills and tuition. However, this support quickly declines early in young adulthood; initially, about 30\% of individuals within this group receive bill support, and close to $15 \%$ receive tuition support. By ages $20-21$, only about $20 \%$ receive parental support for bills, and close to none receive tuition support. A similarly rapid decline occurs for housing support; initially close to three-quarters of individuals report receiving housing assistance, but this drops to less than one-quarter of individuals by ages 22 or 23. Individuals within this group also see themselves as fully financially responsible by age 22 or 23 .

Gradually Independent: Individuals best associated with this group move steadily towards independence over this life stage. This trajectory aligns with the incremental pacing of the transition away from familial support described in prior research (e.g. Furstenberg et al., 
2004; Hartmann and Swartz 2006). Notably, only 23\% of individuals fall within this group ( $\mathrm{N}=$ 396), although normative expectations about this life stage suggest that this is often perceived as the modal experience. Individuals in this group start out receiving support from their families for bills, housing/rent, and tuition for the first few years, but this declines as they age. Supplemental analyses show that among those receiving support for housing, a significant share of individuals received this support via monetary help with rent during their early twenties (53\% at ages 21-22), though both rent and in-kind support have almost completely tapered off by age 26-27, when nearly all respondents in this group are financially responsible for their own housing.

Consistently Supported: Individuals within this final group generally draw on family support for bills, housing, and tuition for the longest duration. By the end of the transition period (ages 26 -27), well over half of the respondents continue to receive parental assistance with bills and housing/rent, and more than a quarter receive parental assistance with tuition. A closer look at housing support shows that parental co-residence, or "doubling up," and rent support serve as substitutes for this group during life stage. During the intermediate years, young adults report lower levels of co-residence with parents, but as shown in Figure 1, average levels of housing support remain unchanged. This is because young adults not living with parents are still receiving financial transfers for housing at these ages. Interestingly, in contrast to this extended duration of family support, those most likely to be in this trajectory group have marked increases in self-reported financial responsibility over time. This suggests that, on average, young adults in this group see themselves as mostly responsible for their finances by ages 26-27, even though they continue to receive multiple kinds of support. Only about 13\% of individuals fall within this final group $(\mathrm{N}=215)$, indicating that an experience of extended financial support during the 
transition to adulthood is limited to a small group of individuals even though this type of experience has been prominently featured in public discourse about the transition to adulthood.

\section{Characteristics of the Multi-Trajectory Groups}

Assessing the four trajectories, it is evident that there is variation in levels of financial independence overall and across types of support received, particularly with respect to the receipt of tuition support. There are also differences in group composition across the trajectories. Using the posterior probabilities of group membership, we assemble weighted average socioeconomic and demographic profiles of the four trajectory groups, the results of which are presented in Table 2. Table 2 reports both group summaries of the time-invariant characteristics used as risk factors in our model as well as additional end-of-survey characteristics that provide a fuller picture of who these young adults are.

There are clear differences in these characteristics across groups. The Consistently Independent group is a racially diverse group of young adults who generally come from households of lower SES, as indicated by their relatively lower levels of parental education (12 years) and their relatively lower levels of high school completion by the start of the survey. Although $60 \%$ of individuals matched to this group reported having attended some postsecondary education, only $44 \%$ completed a four-year degree by the end of the survey period. This group also has the smallest share of student debt holders at ages $27-28$ (28\% of the group reports holding student debt). A significant share of individuals matched to this group have children (57\%) and many are cohabiting or married (23\% and 18\%, respectively). On average, individuals in the Quickly Independent group share many characteristics with Consistently Independent individuals, but two notable differences remain. Those in the Quickly Independent 
group are substantially more likely to have a high school degree at the start of the survey (80\% vs $66 \%, \mathrm{p}<.05$ ), and, at the end of the study period, to be employed ( $85 \%$ vs. $43 \%, \mathrm{p}<.05$ ).

The Gradually Independent and Consistently Supported groups are composed of individuals at the higher end of the socioeconomic spectrum. Average parental education for both groups is over 15 years, suggesting that many of these young adults' parents have at least a college-level education. These two groups are also predominately non-Hispanic white (81\% and 73\%, respectively). The Consistently Supported group is also largely composed of individuals who are single and childless when observed in their late twenties: $81 \%$ are single and only $9 \%$ have children. Among those in the Gradually Independent group, on the other hand, 26\% are married and 17\% have children. Almost all young adults in both groups have pursued and completed four-year college degrees themselves ( $96 \%$ and $98 \%$, respectively). Broadly, patterns and prevalence of familial support for tuition for these two latter trajectories appear to go handin-hand with significantly higher levels of educational attainment for these young adults. Interestingly, the Gradually Independent group has the highest share of individuals with student debt (56\%), while the Consistently Supported group has a similar level of college completion but a lower share of individuals with debt.

We also considered the possibility that the timing of entry into the transition to adulthood relative to the Great Recession might be associated with young adults' financial independence trajectories. The Consistently Supported group has the highest share of individuals (42\%) who turned 18 following the recession. And, although not statistically significantly different from the Quickly Independent group, the magnitude of the share of individuals in the Consistently Independent group is much higher than that of the reference group (37\% vs. 28\%). While it cannot be tested here, this finding suggests that the recession itself might have further stratified 
young adults' receipt of financial support from their parents with respect to socioeconomic status, with higher SES individuals receiving financial support even later into the transition to adulthood and for less advantaged young adults, next-to-no support, save for housing/rent.

\section{The Financial Independence/Financial Security Link}

In the literature on the financial experiences of young adults, financial independence is often seen as a necessary marker of a successful transition to adulthood. However, by focusing on financial independence alone, we ignore other dimensions of young adults' financial wellbeing, namely, their financial security. To address this gap, we turn now to the linkage between independence and security. Descriptively, there are signs that financial security might be closely associated to the timing of financial independence. Our GBTA results show that the trajectories characterized by little financial support from family for bills and tuition (the Consistently and Quickly Independent groups) are much more likely to be matched to individuals from lower SES backgrounds, and much smaller proportions of those in these trajectory groups have completed any post-secondary education by their late twenties than those in the Gradually Independent and Consistently Supported groups. Further, only 43\% of Quickly Independent young adults are employed at the end of the survey period. Conversely, nearly all individuals matched to the Gradually Independent and Consistently Supported not only have some post-secondary education, but obtain four-year college degrees, and have higher levels of parental education. Their trajectories are defined by longer durations of familial financial support. Although financial independence is at times discussed positively within the public discourse, descriptive differences in the composition and experiences of our estimated trajectory groups suggest that the delay of financial independence could be associated with higher levels of financial security. 
To unpack this relationship more directly, we turn to our final analytic step, in which we examine the association between the timing of financial independence and financial security, accounting for end-of-trajectory family and employment characteristics of the young adults. We examine both a subjective measure of financial security, self-reported worry about money, and an objective measure, poverty status, taken at the time the individual is last seen in the survey. We estimate a series of nested regression models that incrementally account for different characteristics and experiences common to the transition to adulthood that might explain the relationship between financial independence trajectory and financial security. Model 1 estimates each measure of financial security as a function of trajectory group membership and two family characteristics that could affect one's financial needs, resources, and security (partnership and parental status at the time last observed). Models 2 and 3 account additionally for employment status at the time at which financial security is measured, as employment likely matters a great deal for both perceived and material financial security. Model 2 adds employment status as a covariate while Model 3 adds an interaction term for employment status and trajectory group. We consider both the mediating and moderating impacts of employment on financial security as we not only expect a positive association between current employment and measures of financial security, but also that employment might matter more for individuals who were financially independent relatively early in young adulthood and thus more reliant on their own wage/salary income. Models are weighted to account for individuals' probability of being matched to each trajectory group. The modal trajectory group of Quickly Independent young adults serves as the reference category. The results of both sets of models are presented in Table 3 and reported as predicted means for level of financial worry and predicted probabilities for poverty status.

[Insert Table 3 about here] 
Worry about Money. Financial worry is a 7-point scale in which higher values indicate higher levels of and more frequent worry about money matters. All groups report moderate levels of financial worry at time last seen; however, some statistically significant and meaningful differences emerge. In the first model, the Consistently Independent group reports the most frequent financial worry (3.97; $\mathrm{p}<.05$ compared to all other groups). The inclusion of employment does reduce the difference in worry between the Consistently and Quickly Independent groups, but employment as a mediating and moderating factor does not significantly impact the variation in financial well-being between the Consistently Independent and the two groups receiving the most support over the transition to adulthood. In Model 3, for instance, in comparing the Consistently Independent and Consistently Supported groups, the mean level of worry among Consistently Independent young adults is approximately 0.4 units or $12 \%$ higher that of the Consistently Supported, who are more likely to have familial support for a longer duration during this life stage. This suggests that those who attain financial independence early in young adulthood are, on average, experiencing financial worry with more frequency.

Poverty Status. Moving to poverty status, we model the likelihood that a young adult's household is below the federal poverty line at time last seen, as a function of the same sets of covariates as in our analyses of financial worry. The first model shows that net of marital and parental status, those who are Consistently Independent are in especially financially precarious standing. These young adults are 2.5 to 3.5 times more likely to be in poverty at time last seen than those who are Consistently Supported and Gradually Independent, respectively. Accounting for employment status in Model 2 and the interaction between employment status and trajectory group in Model 3 has virtually no effect on these associations. 
Young adults with initial familial support but who then become Quickly Independent are also more likely to be in poverty than those who are Gradually Independent and Consistently Supported across all three models. In Model 3, just seven and 10 percent of Gradually Independent and Consistently Supported young adults are likely to be in poverty, but this share doubles for those who are Quickly Independent (20\%). The predicted probability of the Quickly Independent group increases when moving from Model 1 to Model 3 suggesting that employment status is a meaningful moderator in the group-poverty status link for Quickly Independent young adults, but not others. Although the initial predicted probabilities of poverty are significantly lower for those who are Quickly Independent than those who are Consistently Independent in Models 1 and 2, introducing employment as a moderator in Model 3 reduces the difference between these two groups, and the difference is no longer significant.

Together, these two measures of financial security show strong associations with young adults' trajectories of financial support and independence. The Consistently Independent group, comprised of individuals who are most likely to be financially independent early in young adulthood, has both the highest level of financial worry and the highest likelihood of being in poverty at the time last seen. Individuals within this group may have perhaps "achieved" financial independence earlier in young adulthood, but this does not mean that they are financially secure. Similarly, Quickly Independent individuals, though they are marginally less likely to be below the poverty line relative to their Consistently Independent peers, are still more likely to be poor than those who are Gradually Independent and Consistently Supported. Importantly, the Consistently and Quickly Independent groups account for the great majority of the sample (64\%), suggesting that financial insecurity paired with high and relatively early levels of financial independence, is common in young adulthood. Conversely, the experience of relative 
financial security, as defined by lower levels of self-reported worry about money and a lower likelihood of being poor, is shared by only a minority of our sample.

\section{DISCUSSION}

Much research on the transition to adulthood emphasizes the rise of extended receipt of familial financial support during this life stage and interprets this trend as an indicator of delayed adulthood. However, our analyses present a more complex picture of transitions to financial independence and the implications of those experiences for economic security. First, our estimated trajectories of financial support provide descriptive evidence that most young adults continue to move towards financial independence during the transition to adulthood. This is consistent with prior research that indicates that though economic contexts have shifted, agegradated patterns in financial independence appear to have persisted and to be somewhat consistent across groups (Furstenberg et al., 2004; Hartnett et al., 2012). Yet, our results also show that receipt of familial financial support and the assumption of financial independence are not always linearly associated with age. In fact, there is significant socioeconomic and racial/ethnic variation in the shapes of these trajectories, in the ebb and flow of support over the life course, and in receipt and co-occurrence of different types of support, consistent with prior research that finds social group differences in young adults' experiences in the transition to adulthood. Importantly, we find that variation in the types of transitions to financial independence is associated with variation in young adults' financial security in their late twenties.

A clear picture of diverging outcomes and experiences emerges from our two-phase analysis of financial independence and security. Young adults in the Gradually Independent and Consistently Supported groups come from higher socioeconomic status backgrounds, experience 
familial financial support for expenses for longer, and end the study period with higher levels of financial security, despite the fact that a majority of these individuals carry student debt. Conversely, those best aligned with the Consistently Independent group enter young adulthood with lower levels of socioeconomic status and experience virtually immediate financial independence at the age of 18, with minimal, if any support from their families for bills or tuition. In many cases, these individuals continue to co-reside with the parents, perhaps as a costsaving measure. By their mid-to-late twenties, they experience the highest levels of subjective and objective financial insecurity. Their peers in the Quickly Independent group, while experiencing a bit more support from family early in the transition to adulthood, are only marginally less likely to experience financial insecurity in their late twenties. With the vast majority of young adults in these latter two trajectory groups, the new normal for young adults may be financial independence early, followed by later financial insecurity.

These results provide support for two important empirical takeaways related to socioeconomic status. First, young adults from higher socioeconomic backgrounds, as proxied by parents' educational attainment, are more likely to be receiving extended support in the transition to adulthood. Second, net of own and parental education, those who experience extended financial support from parents appear to be more likely to be financially secure at the end of the study period. In contrast, financial support trajectories that include higher proportions of noncollege-going young adults whose parents also did not attend college are characterized by lower levels of or shorter-term familial support. These young adults exhibit higher levels of financial insecurity at the end of the study period. Notably, even after accounting for factors that could plausibly explain variation in financial security, such as employment status, which could improve one's financial wellbeing, we find that observed disparities in financial security across 
groups remain unchanged. This indicates that extended family support may serve as an important buffer against financial hardship for young adults, but that this option may be restricted to those who come from advantaged backgrounds. Further, our models estimating associations between financial independence trajectories and financial security show that although all young adults in our analyses are largely financially independent by the end of the study period, the nature of their transition to that status is at least descriptively predictive of their financial wellbeing.

There are some key limitations to this study. First, due to the data demands of this analytical strategy and necessary sample restrictions, our results are not nationally representative. However, supplemental group-based trajectory analyses using weights to account for probability of selection into the PSID produce nearly-identical trajectory groups, indicating that these trends are not likely to be limited to our analytic sample. Second, we include three measures of financial support from parents to capture a range of types of aid, but there are other financial needs and types of transfers that we are not able to assess (e.g. provision of child care, domestic work). Notably, our study includes tuition support, but we do not know if respondents consider help with outstanding student debt to be tuition support, an item noted as specifically important within the contemporary economic context (Addo et al., 2016; Houle, 2014). Finally, data limitations constrain our ability to explore other specifications of the relationship between age and receipt of financial support. It is plausible, for example, that a cubic specification may better describe the trajectory of support for some; however, these estimations would require more than three repeated measurements, a requirement that would prohibitively reduce our analytic sample.

Despite these limitations, by taking full advantage of the panel design of the PSID data and the detailed item-specific measures of family financial support in the TAS, we are able to comprehensively detail the relationships between young adults' characteristics and multi- 
dimensional indicators of financial independence and security. Our results provide confirmatory evidence of socioeconomic differences in receipt of familial financial support and new insights into the types and timing of financial support received as well as their consequences for financial security in early adulthood. Taking all of our findings in concert, we conclude that the "delay" of financial independence appears not to be indicative of an arrested progression along the life course or necessarily a signal of economic need but a reflection of privilege and family advantage and how it might be leveraged to bolster later financial security for young adults.

As a careful and rich description of real experiences of a diverse sample of young adults in the U.S., our findings may have important policy implications, particularly those that aim to reduce economic inequality. The positive association we find between duration of family support and young adults' financial security, points towards the critical role that private safety nets or family resources can play in shaping young adults' economic well-being. This further suggests that an enhanced public safety net for individuals who cannot rely on family to address financial insecurity may be critical for breaking potential intergenerational transmission of economic insecurity. With an improved understanding of the heterogeneity in financial independence trajectories during this life stage, discussion of transitions to adulthood can be more nuanced and provide useful information to policymakers and practitioners concerned about the economic lives of young adults, who, holding themselves and their families to age norms related to financial responsibilities and independence, may also be exposing themselves to contexts of financial insecurity. 


\section{REFERENCES}

Addo, F. R., J. N. Houle, \& D. Simon. (2016). Young, black, and (still) in the red: Parental wealth, race, and student loan debt. Race and Social Problems, 8(1), 64-76. doi: 10.1007/s12552-016-9162-0

Browne, I., \& J. Misra. (2003). The intersection of gender and race in the labor market. Annual Review of Sociology 29, 487-513. doi:10.1146/annurev.soc.29.010202.100016

Danzinger, S., \& Ratner, D. (2010). Labor market outcomes and the transition to adulthood. The Future of Children, 20(1), 133-158.

Engemann, K. \& H. J. Wall. (2009). The effects of recessions across demographic groups. Federal Reserve Bank of St. Louis. Retrieved from http://papers.ssrn.com/sol3/papers.cfm?abstract_id=1490041.

Fingerman, K. L., Y.-P. Cheng, E. D. Wesselmann, S. Zarit, F. Furstenberg, \& K. S. Birditt. (2012). Helicopter parents and landing pad kids: Intense parental support of grown children. Journal of Marriage and Family, 74(4), 880-896. doi:10.1111/j.17413737.2012.00987.x

Fingerman, K. L., K. Kim, E. M. Davis, F. F. Furstenberg, K. S. Birditt, and S. H. Zarit. (2015). 'I'll give you the world': Socioeconomic differences in parental support of adult children. Journal of Marriage and Family, 77(4), 844-65. doi:10.1111/jomf.12204

Fligstein, N. \& A. Goldstein. (2015). The emergence of a finance culture in American households, 1989-2007. Socio-Economic Review, 13(3), 575-601. doi:10.1093/ser/mwu035

Furstenberg, F. F. (2010). On a new schedule: Transitions to adulthood and family change. The Future of Children, 20(1), 67-87. doi:10.1353/foc.0.0038

Furstenberg, F. F., S. Kennedy, V. C. McLoyd, R. G. Rumbaut, \& R. A. Settersten, Jr. (2004). Growing up is harder to do. Contexts, 3(3), 33- 41.

Gonzales, R. G. (2011). Learning to be illegal: Undocumented youth and shifting legal contexts in the transition to adulthood. American Sociological Review 76(4), 602-619. doi: $10.1177 / 0003122411411901$

Hacker, J. S. (2006). The great risk shift: The new economic insecurity and the decline of the American dream. United Kingdom: Oxford University Press.

Hamilton, L. (2016). Parenting to a degree: How family matters for college women's success. Chicago: University of Chicago Press. 
Hardaway, C. R. \& V. C. McLoyd. (2009). Escaping poverty and securing middle class status: How race and socioeconomic status shape mobility prospects for African Americans during the transition to adulthood. Journal of Youth and Adolescence 38:242-256. doi: 10.1007/s10964-008-9354-z

Hartmann, D. \& T. T. Swartz. (2006). The new adulthood? The transition to adulthood from the perspective of transitioning young adults. Advances in Life Course Research 11:253-286. doi: 10.1016/S1040-2608(06)11010-2

Hartnett, C. S., F. F. Furstenberg, K. S. Birditt, and K. L. Fingerman. (2012). Parental support during young adulthood: Why does assistance decline with age? Journal of Family Issues, 34(7), 975-1007. doi:10.1177/0192513X12454657

Houle, J. N. (2014). A generation indebted: Young adult debt across three cohorts. Social Problems, 61(3), 448-465. doi:10.1525/sp.2014.12110

Houle, J. N. \& C. Warner. (2017). Into the red and back to the nest? Student debt, college completion, and returning to the parental home among young adults. Sociology of Education, 90(1), 89-108. doi:10.1177/0038040716685873

Johnson, M. K. \& J. Benson. (2012). The implications of family context for the transition to adulthood. In A. Booth, S. L. Brown, N. S. Landale, W. D. Manning, and S. M. McHale (Eds.), Early adulthood in a family context, national symposium on family issues (pp. 87 - 103). New York, NY: Springer.

Jones, B. L. \& D. S. Nagin. (2013). A note on a Stata plugin for estimating group-based trajectory models. Sociological Methods \& Research, 42(4), 608-13. doi:10.1177/0049124113503141

Joint Center for Housing Studies of Harvard University. (2016). The state of the nation's housing. Cambridge, MA. Retrieved from http://www.jchs.harvard.edu/sites/jchs.harvard.edu/files/jchs_2016_state_of_the_nations _housing_lowres.pdf.

Killewald, A. (2013). Return to being black, living in the red: A race gap in wealth that goes beyond social origins. Demography, 50(4),1177-1195. doi:10.1007/s13524-012-0190-0

Kim, C.H. \& C. R. Tamborini. (2012). Response error in earnings: An analysis of the survey of income and program participation matched with administrative data. Sociological Methods \& Research, 43(1), 39-72. doi:10.1177/0049124112460371

Lee, J.C. \& J. T. Mortimer. (2009). Family socialization, economic self-efficacy, and the attainment of financial independence in early adulthood. Longitudinal and Life Course Studies, 1(1), 45-62. 
López Turley, R. N. \& M. Desmond. 2011. Contributions to college costs by married, divorced, and remarried parents.” Journal of Family Issues 32(6), 767-790. doi:

10.1177/0192513X10388013

Maroto, M. (2017). When the kids live at home: Coresidence, parental assets, and economic insecurity. Journal of Marriage and Family, 79(4), 1041-1059. doi:10.1111/jomf.12407

Moen, P. \& E. Wethington. (1992). The concept of family adaptive strategies. Annual Review of Sociology, 18, 233-51. doi:10.1146/annurev.so.18.080192.001313

Mortimer, J. T, M. Kim, J. Staff, \& M. Vuolo. (2016). Unemployment, parental help, and selfefficacy during the transition to adulthood. Work and Occupations, 43(4), 434-465. doi:10.1177/0730888416656904

Mykyta, L. \& S. Macartney. (2012). Sharing a household: Household composition and economic well-being: 2007-2010. Current Population Report P60-242. Washington, DC: U.S. Census Bureau.

Nagin, D. S. (2005). Group-based modeling of development. Cambridge, MA: Harvard University Press.

Nagin, D. S., B. L. Jones, V. Lima Passos, \& R.E. Tremblay. (2016). Group-based multitrajectory modeling. Statistical Methods in Medical Research, 0(0), 1-9. doi:10.1177/0962280216673085

Newman, K. S. (1995). No shame in my game: The working poor in the inner city. New York, NY: Vintage.

Osgood, D. W., E. M. Foster, \& M. E. Courtney. (2010). Vulnerable populations and the transition to adulthood. The Future of Children 20(1), 209-229.

Pager, D. (2003). The mark of a criminal record. American Journal of Sociology 108(5), 937975. doi:10.1086/374403

Pilkauskas, N. V., I. Garfinkel, \& S. S. McLanahan. (2014). The prevalence and economic value of doubling up. Demography, 51(5), 1667-76. doi:10.1007/s13524-014-0327-4

Sandberg-Thoma, S. E., A. R. Snyder, \& B. J. Jang. (2015). Exiting and returning to the parental home for boomerang kids. Journal of Marriage and Family, 77(3), 806-818.

doi:10.1111/jomf.12183

Silva, J. M. (2012). Constructing adulthood in an age of uncertainty. American Sociological Review, 77(4), 505-22. doi:10.1177/0003122412449014 
Sironi, M. \& F. F. Furstenburg. (2012). Trends in the economic independence of young adults in the United States: 1973 - 2007. Population and Development Review, 38(4), 609-630. doi: 10.1111/j.1728-4457.2012.00529.x

Suitor, J. J., \& K. Pillemer. 2006. Choosing daughters: Exploring why mothers favor adult daughters over sons. Sociological Perspectives 49(2), 139-161. doi:10.1525/sop.2006.49.2.139

Survey Research Center. (2017). Panel Study of Income Dynamics, public use dataset. University of Michigan: Survey Research Center, Institute for Social Research.

Survey Research Center. (2015). PSID Transition Into Adulthood Supplement 2015 User Guide. University of Michigan: Survey Research Center, Institute for Social Research.

Swartz, T. T. (2009). Intergenerational family relations in adulthood: patterns, variations, and implications in the contemporary United States. Annual Review of Sociology, 35, 191212. doi:10.1146/annurev.soc.34.040507.134615

Swartz, T. T., M. Kim, M. Uno, \& J. T. Mortimer. (2011). Safety nets and scaffolds: parental support in the transition to adulthood. Journal of Marriage and Family, 73(2), 414-429. doi:10.1111/j.1741-3737.2010.00815.x

U.S. Department of Education. (2016). Educational attainment of young adults. In The condition of education 2016. Washington, DC: National Center for Education Statistics 2016-144.

van der Zwan, N. (2014). Making sense of financialization. Socio-Economic Review, 12(1), 99129. doi:10.1093/ser/mwt020

Warren, J. R., L. Luo, A. Halpern-Manners, J. M. Raymo, \& A. Palloni. (2015). Do different methods for modeling age-graded trajectories yield consistent and valid results? American Journal of Sociology, 120(6), 1809-56. doi:10.1086/681962

Wasserman, L. (2000). Bayesian model selection and averaging. Journal of Mathematical Psychology, 44(1), 92-107. doi: 10.1006/jmps.1999.1278

Waters, M. C., P. J. Carr, M. Kefalas, \& J. A. Holdaway (Eds). (2011). Coming of age in America: The transition to adulthood in the twenty-first century. Berkeley, CA: University of California Press.

Wightman, P., R. Schoeni, \& K. Robinson. (2012). Familial financial assistance to young adults. National Poverty Center Working Paper Series 12-10. Ann Arbor, MI: National Poverty Center, University of Michigan. 


\section{MethodologicAl APPENDiX}

Group-based trajectory models require the researcher to specify the number of trajectories and the polynomial orders of the trajectories. We make these decisions using theory and statistical checks, following a modified version of the two-phase selection process outlined by Nagin (2005) for the multi-trajectory analysis that enables our simultaneous examination multiple types of financial support and responsibility (Nagin et al., 2016). All analyses were conducted using the Traj Stata package developed specifically for conducting group-based trajectory analysis (Jones and Nagin, 2013).

In the first phase, we identify the appropriate number of trajectories for each of our four financial responsibility measures. The trajectories we then model are approximations. The objective here is to reduce the data to identify types of trajectories similarities in financial support and responsibility experiences across individuals, summarizing types of trajectories, while continuing to highlight substantively important differences that emerge. We compare models that approximate $2,3,4$, and 5 trajectory groups for each of the financial responsibility items and identify the appropriate number of groups for each measure by comparing Bayesian Information Criterions (BIC) for each model (Wasserman, 2000). Based on model assessments for each measure, we established that the best fit models for all measures identified at least 4 trajectory groups; for the measures of parental support, 4-group models yielded the best fit (lowest BIC). In the case of self-reported financial responsibility, a 5-trajectory group solution fit best.

However, in order to run a multi-trajectory analysis that considers together parental support for bills, tuition, rent, and self-reported financial responsibility, the model specifications must include the same number of trajectory groups for each of the four dependent variables 
(Nagin et al., 2016). This final specification required us to modify the model for self-reported financial responsibility. While the best model for financial responsibility was a 5-group model, we elect to instead use the second-best model (4 trajectory groups) because the best models for tuition, rent, and financial responsibility were 4-group models.

In the second phase, with the number of trajectory groups identified, we turned to the task of identifying the appropriate polynomial order for each trajectory. To do so, we relied on the extant literature and the hypotheses outlined in our paper. Importantly, we expected to see clear distinctions in the paths of the trajectories that correspond to the SES factors that will impact the "risk" of financial support from parents and for one’s self-reported financial responsibility:

On one end of the spectrum, we expected that there would be a group of individuals who by-and-large never receive support from their families because it is too costly for their families. In this case, individuals would follow a stable, zero-order trajectory, with consistently few people receiving support for bills, tuition, or housing across this life stage. On the other end, we expected that there might be a group of individuals for whom parental support is consistently high across this life stage. In this case, individuals would follow a stable zero-order trajectory where many people within the group receive parental support at each wave. Finally, based on the research consensus that many young adults do become increasingly independent across this life stage, we expected that the remaining two trajectories would start out with the majority of individuals receiving parental support for bills, tuition and/or rent, but that this support declines in a potentially non-linear fashion as they age. We expected the two trajectory groups to vary in the proportion of individuals receiving support at each age, and for the trajectories to have different slopes. 
Echoing this, the analysis for self-reported financial independence should yield a trajectory of consistently high levels of financial responsibility for one group (that could mirror those who consistently never receive parental support), with another set of quadratic trajectories of increasing financial independence over this life stage, albeit at varying levels and slopes. Finally, we expected a fourth trajectory of relatively low rankings of self-reported financial independence as these individuals may continue to rely on family support in all waves.

In Phase 2, we begin with the 4-group model identified in the first stage of analysis and set our initial polynomial order sequence as $(0,2,2,0)$. We then again use BIC to identify the best functional form for each financial responsibility indicator, where 0 represents a zero-order polynomial and 2 represents a quadratic. We compared this theoretical set against other combinations of orders for each of the four financial responsibility models, and selected the best fitting polynomial orders using BIC comparison. The best order of trajectories is as follows for each of the four measures, and we use these to proceed with our multi-trajectory analysis:

Support with Bills: $(0,2,2,1)$

Support with Housing/Rent: $(0,2,1,0)$

Support with Tuition: $(0,1,2,2)$

Self-Assessed Financial Responsibility: $(0,2,1,1)$

The multi-trajectory model is fitted with the above specifications. As illustrated in Figure 1, this final model produces four trajectory groups for overall financial independence that are defined by a set of trajectories for each of the individual financial independence markers. 
Appendix B. Multi-Trajectory Model Results: Risk Factors

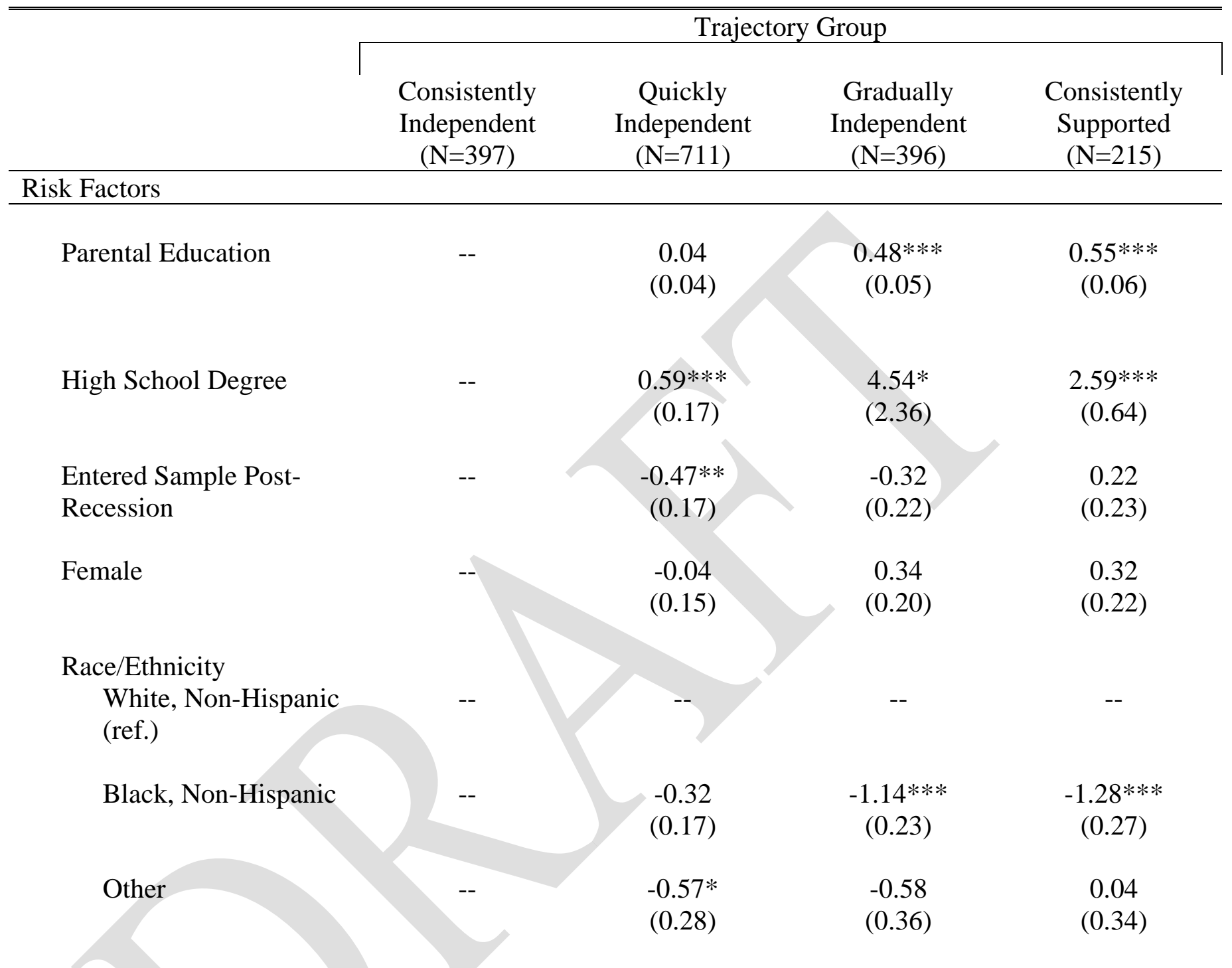

Notes: $\mathrm{N}=1,719$. This table provides the impacts of the five risk factors on the probability of membership in each trajectory group, relative to the Consistently Independent group. For instance, the probability of membership in either the Gradually Independent or Consistently Supported groups, relative to the Consistently Independent group, is significantly lower if the individual is black $(\mathrm{p}<.001)$. 
Table 1. Sample Characteristics: PSID Transition to Adulthood Supplement, 2005-2015

Mean or Proportion

Parents' Educational Attainment

13.97

(2.78)

High School Degree/Equivalent

0.80

Female

0.53

Race/Ethnicity

White, Non-Hispanic

0.63

Black, Non-Hispanic

0.17

Other

0.20

Age at 1st Observation

18.64

Age at Last Observation

24.94

Number of Years Spanned

6.30

(1.48)

Financial Responsibility and Security

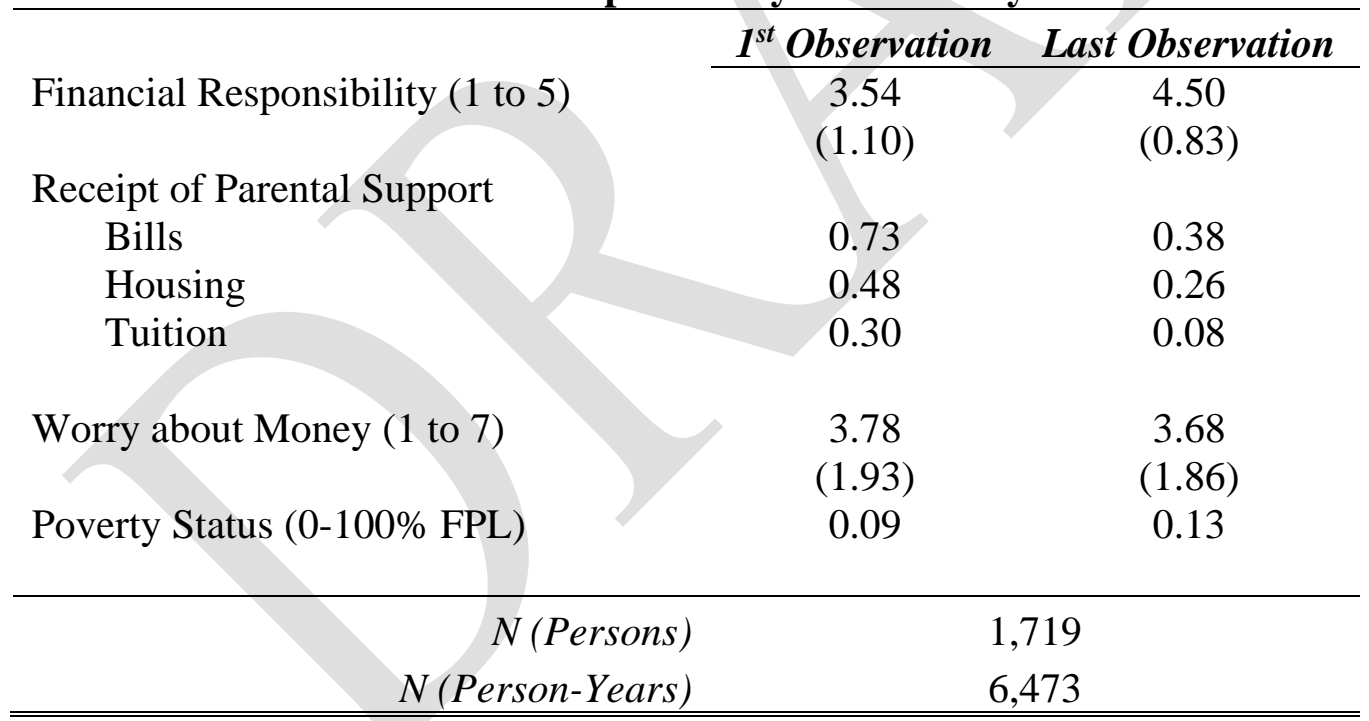

Note: Estimates are weighted. 
Table 2. Group Characteristics from Multi-Trajectory Model

\begin{tabular}{|c|c|c|c|c|}
\hline & \multicolumn{4}{|c|}{ Trajectory Group Means (SD) and Proportions } \\
\hline & $\begin{array}{c}\text { Consistently } \\
\text { Independent } \\
(\mathrm{N}=397)\end{array}$ & $\begin{array}{c}\text { Quickly } \\
\text { Independent } \\
(\mathrm{N}=711)\end{array}$ & $\begin{array}{l}\text { Gradually } \\
\text { Independent } \\
(\mathrm{N}=396)\end{array}$ & $\begin{array}{c}\text { Consistently } \\
\text { Supported } \\
(\mathrm{N}=215)\end{array}$ \\
\hline Risk Factors & & & & \\
\hline Parental Education & $\begin{array}{l}12.31 \\
(2.83)\end{array}$ & $\begin{array}{l}13.00 \\
(2.82)\end{array}$ & $\begin{array}{l}15.53 * \\
(1.90)\end{array}$ & $\begin{array}{c}15.59 * \\
(1.78)\end{array}$ \\
\hline High School Degree & $0.66^{*}$ & 0.80 & $1.00 *$ & $0.97 *$ \\
\hline Entered Sample Post-Recession & 0.37 & 0.28 & 0.31 & $0.42 *$ \\
\hline Female & 0.52 & 0.48 & 0.58 & 0.50 \\
\hline $\begin{array}{l}\text { Race/Ethnicity } \\
\text { White, Non-Hispanic }\end{array}$ & 0.43 & 0.55 & $0.81 *$ & $0.73^{*}$ \\
\hline Black, Non-Hispanic & 0.28 & 0.24 & $0.07^{*}$ & $0.04 *$ \\
\hline Other & 0.29 & 0.21 & $0.12 *$ & 0.22 \\
\hline End-of-Trajectory Characteristics & & & & \\
\hline Age at Final Observation & $\begin{array}{l}24.85 \\
(1.43)\end{array}$ & $\begin{array}{l}25.10 \\
(1.41)\end{array}$ & $\begin{array}{l}25.07 \\
(1.41)\end{array}$ & $\begin{array}{l}24.67 * \\
(1.61)\end{array}$ \\
\hline Any Post-Secondary Education & 0.60 & 0.69 & $0.98^{*}$ & $0.99 *$ \\
\hline $\begin{array}{l}\text { Completed 4-Year College } \\
\text { Degree }\end{array}$ & 0.44 & 0.54 & $0.96 *$ & $0.98^{*}$ \\
\hline Has Student Debt & 0.28 & 0.36 & $0.56^{*}$ & 0.44 \\
\hline $\begin{array}{l}\text { Relationship Status } \\
\text { Single }\end{array}$ & 0.59 & 0.51 & 0.57 & $0.81^{*}$ \\
\hline Cohabiting & 0.18 & 0.21 & 0.17 & $0.09 *$ \\
\hline Married & 0.23 & 0.28 & 0.26 & $0.11 *$ \\
\hline Parental Status (Has Child) & 0.57 & 0.42 & $0.17^{*}$ & $0.04 *$ \\
\hline Employment Status & $0.43^{*}$ & 0.85 & 0.82 & $0.62 *$ \\
\hline
\end{tabular}

Notes: ${ }^{*} \mathrm{p}<0.05$; the modal group, Quickly Independent, is the reference group. All estimates are weighted to account for both PSID oversampling of low income households, and differential probabilities of being within each trajectory group. 
Table 3. Models of Financial Security: Worry about Money and Poverty Status

\begin{tabular}{|c|c|c|c|}
\hline & Model 1 & Model 2 & Model 3 \\
\hline \multicolumn{4}{|l|}{$\begin{array}{l}\text { Level of Financial Worry } \\
\text { (1 = Rarely Worry, } 7 \text { = Daily Worry) }\end{array}$} \\
\hline A: Consistently Independent & $\begin{array}{l}3.97 \mathrm{~B}, \mathrm{C}, \mathrm{D} \\
(0.10)\end{array}$ & $\begin{array}{c}3.92 \mathrm{C}, \mathrm{D} \\
(0.10)\end{array}$ & $\begin{array}{c}3.93 \mathrm{C}, \mathrm{D} \\
(0.11)\end{array}$ \\
\hline B: Quickly Independent & $\begin{array}{l}3.63 \mathrm{~A} \\
(0.07)\end{array}$ & $\begin{array}{l}3.67 \\
(0.07)\end{array}$ & $\begin{array}{c}3.71 \\
(0.08)\end{array}$ \\
\hline C: Gradually Independent & $\begin{array}{l}3.48 \mathrm{~A} \\
(0.09)\end{array}$ & $\begin{array}{l}3.52 \mathrm{~A} \\
(0.09)\end{array}$ & $\begin{array}{l}3.53 \mathrm{~A} \\
(0.09)\end{array}$ \\
\hline D: Consistently Supported & $\begin{array}{l}3.52 \mathrm{~A} \\
(0.12)\end{array}$ & $\begin{array}{l}3.51 \mathrm{~A} \\
(0.12)\end{array}$ & $\begin{array}{l}3.52 \mathrm{~A} \\
(0.12)\end{array}$ \\
\hline \multicolumn{4}{|l|}{$\begin{array}{l}\text { Poverty Status } \\
(<100 \% \text { Federal Poverty Line })\end{array}$} \\
\hline A: Consistently Independent & $\begin{array}{l}0.27 \text { в, C, D } \\
(0.02)\end{array}$ & $\begin{array}{c}0.26 \mathrm{~B}, \mathrm{C}, \mathrm{D} \\
(0.02)\end{array}$ & $\begin{array}{c}0.25 \mathrm{C}, \mathrm{D} \\
(0.02)\end{array}$ \\
\hline B: Quickly Independent & $\begin{array}{c}0.18 \mathrm{~A}, \mathrm{C}, \mathrm{D} \\
(0.01)\end{array}$ & $\begin{array}{c}0.20 \mathrm{~A}, \mathrm{C}, \mathrm{D} \\
(0.02)\end{array}$ & $\begin{array}{c}0.20 \mathrm{C}, \mathrm{D} \\
(0.02)\end{array}$ \\
\hline C: Gradually Independent & $\begin{array}{l}0.08 \mathrm{~A}, \mathrm{~B} \\
(0.01)\end{array}$ & $\begin{array}{c}0.08 \mathrm{~A}, \mathrm{~B} \\
(0.02)\end{array}$ & $\begin{array}{c}0.07 \mathrm{~A}, \mathrm{~B} \\
(0.01)\end{array}$ \\
\hline D: Consistently Supported & $\begin{array}{c}0.10 \mathrm{~A}, \mathrm{~B} \\
(0.02)\end{array}$ & $\begin{array}{c}0.10 \mathrm{~A}, \mathrm{~B} \\
(0.02)\end{array}$ & $\begin{array}{c}0.10 \mathrm{~A}, \mathrm{~B} \\
(0.02)\end{array}$ \\
\hline Family Characteristics & $\mathrm{X}$ & $\mathrm{X}$ & $\mathrm{X}$ \\
\hline Employment Status & & $\mathrm{X}$ & $\mathrm{X}$ \\
\hline Employment Status x Group & & & $\mathrm{X}$ \\
\hline
\end{tabular}

Notes: $\mathrm{N}=1,719$ for all models. Superscript letters indicate statistically significant differences from other groups at $\mathrm{p}<.05$, using pairwise comparisons. All estimates are weighted to account for differential probabilities of being in each group. Family characteristics and employment status are measured at the last wave observed, the same time at which financial security is measured. Standard errors are reported in parentheses. 
Figure 1. Multi-Trajectory Model FOr Overall FinANCIAL INDEPENDENCE
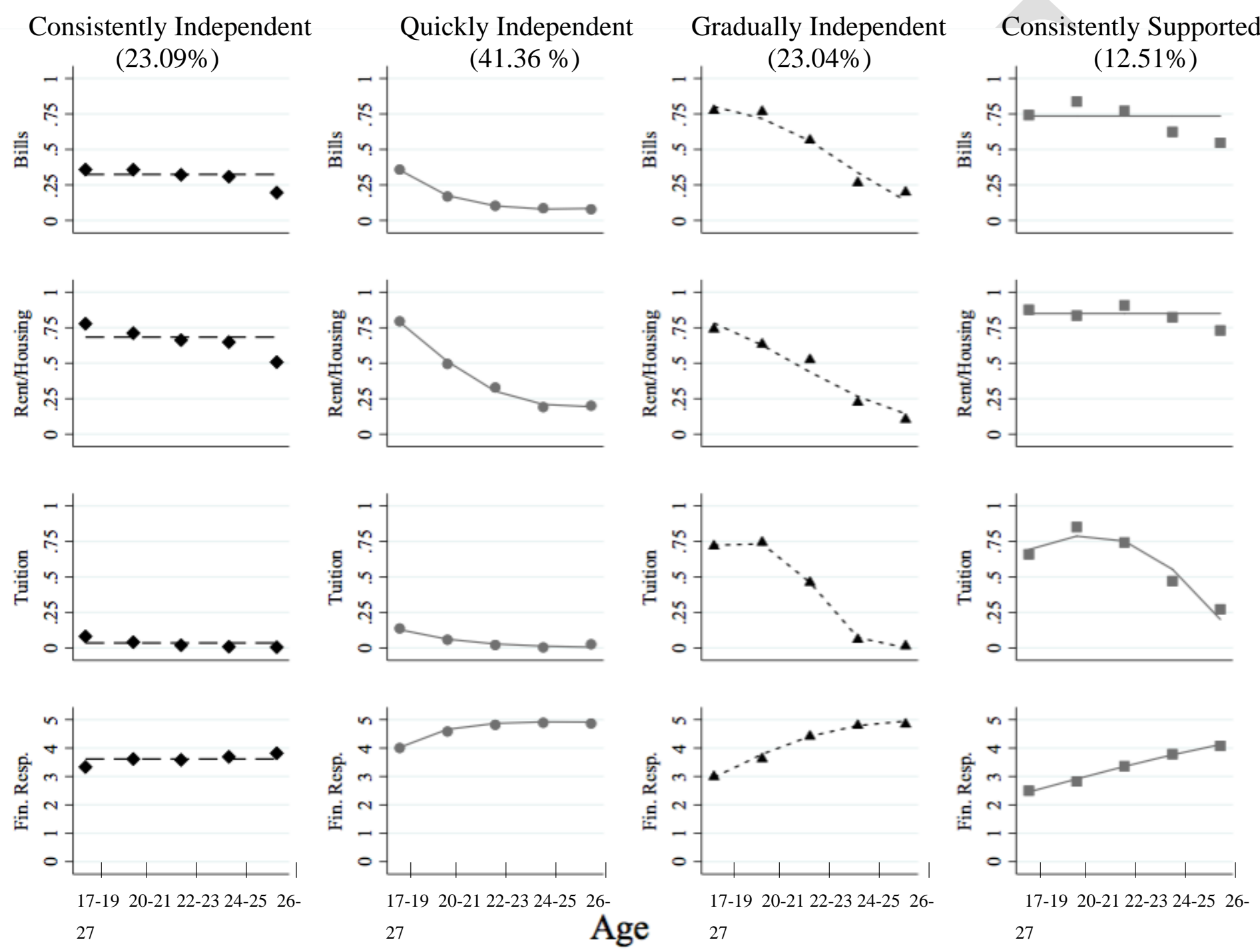

Notes: $\mathrm{N}=1,719$ individuals with 6,473 observations. Graphs plot the estimated trajectories (lines) for each group with the observed group means (markers). 